\title{
Optimum Combination of Reversed-Phase Column Type and Mobile-Phase Composition for Gradient Elution Ion-Pair Chromatography of Amino Acids
}

\author{
Yukio Yokoyama ${ }^{\dagger}$, Tomoo Amaki, Sohei Horikoshi and Hisakuni Sato \\ Laboratory of Analytical Chemistry, Faculty of Engineering, Yokohama National University, \\ Tokiwadai, Hodogaya, Yokohama 240, Japan
}

\begin{abstract}
An optimum combination of reversed-phase column type and eluent composition was selected for the practical separation of amino acids by gradient elution ion-pair chromatography. A butyl-silica (C4) column with a concentration gradient elution between $5 \mathrm{mM} \mathrm{SDS}-10 \mathrm{mM} \mathrm{HClO}_{4}$ solution and acetonitrile provided the best resolution for amino acids. The C4 column was most adequate for reducing the chromatographic cycle time including the column regeneration. The use of $5 \mathrm{mM}$ SDS as ion-pairing agent in relatively low concentration is economical and practical for replicate and routine analyses. The developed HPLC system is applicable to the analysis of organic cations at low cost.
\end{abstract}

Keywords Ion-pair high-performance liquid chromatography, gradient elution, sodium dodecylsulfate, sodium octanesulfonate, amino acid, organic cation

The analysis of amino acids using high-performance liquid chromatography (HPLC) techniques is very important in biological and biomedical laboratories. The widely accepted methods, so-called amino acid analyzers, are mainly based on cation-exchange separation followed by postcolumn derivatization such as $o$-phthalaldehyde (OPA) fluorescence derivatization $^{1-3}$ and reversed-phase separation of precolumn derivatization products such as OPA derivatives. ${ }^{4-6}$ Such instruments designed and specialized for routine use are of course commercially available, but they are expensive and unavailable for general purposes.

As a relatively fast and simple procedure for amino acid analysis, gradient elution ion-pair chromatography techniques have also been proposed ${ }^{7-9}$, but these have been unpopular so far owing to their poor reproducibility in replicate chromatography. To overcome the poor reproducibility, auto-samplers are sometimes employed for timed sample injections between the gradient runs. In any case, however, it is difficult to obtain reproducible retention times unless we know the real column reequilibration. It is known that the gradient ion-pair HPLC needs a long time for regenerating the reversed-phase column to the initial ionic equilibrium between the stationary and mobile phases, requiring more than 15-20 column volumes of initial solvent. ${ }^{10}$ Patthy ${ }^{11}$ has experimentally estimated the reequilibration of a reversed-phase column between gradient runs, by finding the plateau of retention times of standard analytes after several different regeneration times. Even

\footnotetext{
† To whom correspondence should be addressed.
}

by this method, however, we can not know the real reequilibration finish.

We recently have developed a time and cost efficient HPLC method for amino acid analysis using dual-mode gradient ion-pair chromatography. ${ }^{12}$ A butyl-silica (C4) reversed-phase column and a sodium dodecylsulfate (SDS)/perchloric acid/acetonitrile eluent system with a simultaneous concentration and flow-rate gradient elution technique has been efficiently applied. In the process of developing the above method, we have discovered that the ghost peak appearing on the UV baseline is due to the completion of reequilibration of the separation column after the acetonitrile gradient elutions. In the previous paper, however, the optimum combination of reversed-phase column and mobile phase composition has not been discussed in any detail. The regeneration cycle of gradient ion-pair chromatography depends on both the degree of hydrophobicity of the stationary phase and that of the ion-pairing agent in the mobile phase. Naturally the combination of the two phases affects the separation and retention of the amino acids.

Knox ${ }^{13}$ and co-workers have detailed a theoretical mechanism of the adsorption of ion-pairing agent to the stationary phase. In practice, however, it seems to be difficult to estimate theoretically the retention by different columns, because all parameters for the theoretical calculations must be different for the different columns.

In general, reversed-phase columns show poor reproducibility in their retention power. Even within one brand from the same supplier, reversed-phase columns of different product lots often provide different 
retention times under the same eluting condition. This seems to be due to the unevenness in hydrophobicity (carbon loadings) of the chemically bonded silica-gel packing. Therefore it is thought to be necessary to find experimentally the column hydrophobicity adequate for the intended ion-pair separation.

This paper presents a practical technique to find optimum combinations of reversed-phase column and mobile phase composition in the gradient ion-pair chromatography of amino acids.

\section{Experimental}

\section{Instrumentation}

The HPLC system consisted of an ERC (Tokyo, Japan) Model 3510 solvent degasser, two Shimadzu (Kyoto, Japan) LC-9A pumps with a high-pressure solvent mixer for two liquids, a modified Toyo (Tokyo, Japan) FI-45 incubator as column oven equipped with a Rheodyne (Cotati, CA, USA) Model 7125 syringeloading sample injector with a $100-\mu l$ sample loop, a Shimadzu SPD-10A UV spectrophotometric detector and a Hitachi (Tokyo, Japan) D-2500 integrator. A Rheodyne 7335 column inlet filter was placed between the injector and the separation column. The detection wavelength was $210 \mathrm{~nm}$; the oven temperature was $30^{\circ} \mathrm{C}$; and the sample injection volume was $20 \mu \mathrm{l}$, throughout the experiment.

\section{Materials and reagents}

Three GL Sciences (Tokyo, Japan) Inertsil C4, C8, and ODS-2 reversed-phase HPLC columns (150 $\mathrm{mm} \times 4.6$ $\mathrm{mm}$ i.d. each) were used. The particle size of each packing was $5 \mu \mathrm{m}$ and the nominal theoretical plate number $(N)$ was almost the same: 13000 per each column. The nominal carbon loadings of the respective columns were $7.5,10.5$, and $18.5 \%$. A GL Sciences Inertsil C4 column of $250 \mathrm{~mm}$ in length (4.6 mm i.d., $N=22000$ ) was also used.

Perchloric acid of super special grade and acetonitrile of HPLC grade were purchased from Wako (Osaka, Japan), and SDS of $98 \%$ purity was purchased from Aldrich (Milwaukee, WI, USA). Sodium octanesulfonate (SOS) and sodium pentanesulfonate (SPS) both of ion-pair chromatography grade were purchased from Tokyo Kasei (Tokyo, Japan). The standard amino acids were purchased from Sigma (St. Louis, MO, USA) or Wako. These reagents were used without further purification. Deionized water through an ORGANO (Tokyo, Japan) Model G-10 mixed-bed ion-exchange cartridge system was purified by a Nihon Millipore (Tokyo, Japan) MILLI-Q Labo water purification system just before use.

\section{Gradient elution}

A high-pressure binary gradient elution (mobile phase $A$ and $B$ ) was executed in each case using two kinds of ion-pairing agents. Mobile phase A consisting of the ion-pairing agent and acid in different concentrations examined were as follows: 1,5 and $10 \mathrm{mM}$ SDS/ 1,5 and $10 \mathrm{mM} \mathrm{HClO}_{4}$ in nine combinations; 10,50 and $100 \mathrm{mM} \mathrm{SOS} / 10 \mathrm{mM} \mathrm{HClO}_{4}$ in three combinations. The mobile phase $B$ was $100 \%$ acetonitrile. In the case of SDS, the mixing ratio of the mobile phases $A$ and $B$ was varied linearly from $A / B=85 / 15$ at 0 min to $80 / 20$ at $20 \mathrm{~min}$ and consecutively to $65 / 35$ at $50 \mathrm{~min}$. In the case of SOS, the linear gradient from $A / B=100 / 0$ at 0 min to $75 / 25$ at $50 \mathrm{~min}$ was programmed. In both cases, when returning to their initial $\mathrm{A} / \mathrm{B}$ ratios at $55 \mathrm{~min}$, the initial mobile phase was kept running to the baseline system peak $^{12}$ indicating the completion of column regeneration. The flow rate was kept constant at $1.0 \mathrm{ml} / \mathrm{min}$ throughout the experiment.

\section{Regeneration time}

The ion-pairing agent adsorbed into the reversedphase column is desorbed from the stationary phase during each gradient run, due to the increase in organic concentration in the mobile phase. After each gradient run, the completion of column regeneration to the initial ionic equilibrium can be determined by observing the baseline system peak as described in a previous paper. ${ }^{12}$ The regeneration time corresponds to the retention time of the system peak minus $55 \mathrm{~min}$ in this work. Consequently, the "re-adsorption" amount of SDS to the stationary phase between the gradient runs can be estimated by the product of the initial concentration of the ion-pairing agent (e.g., $4.25 \mathrm{mM}$ SDS from $85 \%$ of $5 \mathrm{mM}$ SDS in mobile phase A) and the regeneration volume (time $\times$ flow rate).

\section{Results and Discussion}

\section{Optimization of SDS eluent system}

In ion-pair HPLC, regeneration of the analytical column to its initial condition after gradient elution is essential to obtain reproducible and reliable results. It is naturally considered that the regeneration time depends on the hydrophobicity of both the reversedphase column packings and the ion-pairing agent and also on the concentration of the acid and the ion-pairing agent in the mobile phase. Figure 1 shows relationships between the re-adsorption amount of SDS and the degree of column hydrophobicity referred to as their nominal carbon loadings. Although the real carbon loadings of the columns may be different from the nominal values, the $\mathrm{C} 18$ (ODS) column must be the most hydrophobic one in general. Contrary to this, the re-adsorption amount of SDS for the C18 column was less than that for the $\mathrm{C} 8$ column. Especially under the $10 \mathrm{mM} \mathrm{SDS/}$ $5 \mathrm{mM} \mathrm{HClO}_{4}$ condition, the re-adsorption amount for the $\mathrm{C} 18$ column was nearly equal to that for the $\mathrm{C} 4$ column. This result suggests that the inconsistent decrease in the SDS amount can originate from insufficient solvation between the higher lipophilic $\mathrm{C} 18$ packings and the water-rich $(85 \mathrm{v} / \mathrm{v} \%)$ acetonitrile eluent. In other words, the octadecyl chain on the silica- 


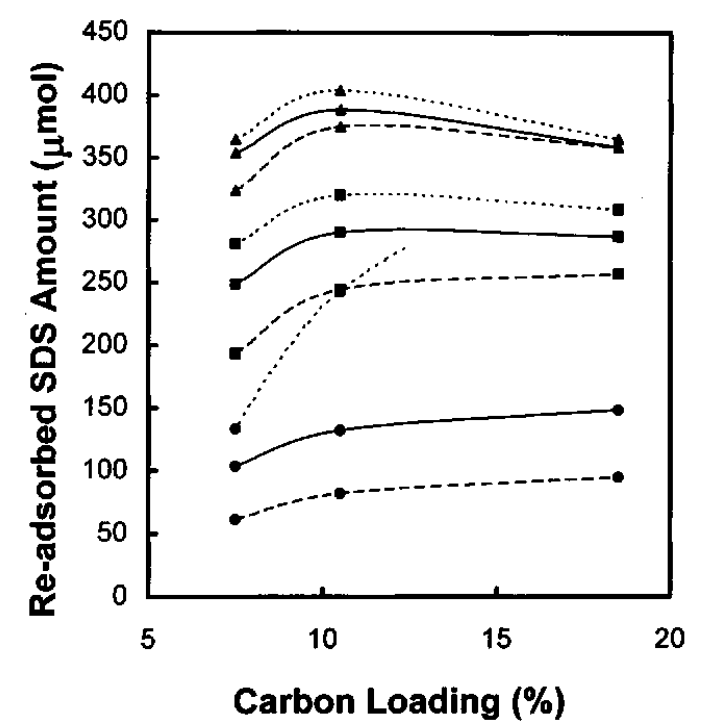

Fig. 1 Relationships between nominal carbon loading of stationary phase and re-adsorption amount of SDS to the stationary phase. Symbols represent SDS concentration as: $\bigcirc, 1 \mathrm{mM} ; \square, 5 \mathrm{mM} ; \Delta, 10 \mathrm{mM}$, and lines represent $\mathrm{HClO}_{4}$ concentration as: broken, $1 \mathrm{mM}$; solid, $5 \mathrm{mM}$; dotted, $10 \mathrm{mM}$.

gel surface can partially soak into the water-rich mobile phase owing to the existence of a repelling effect between the two phases, so that the adsorption site for SDS must in fact be decreased.

Figure 2 shows relationships between $\mathrm{HClO}_{4}$ concentration in the mobile phase and regeneration time. The increase in concentration of $\mathrm{HClO}_{4}$, i.e. the decrease in $\mathrm{pH}$, led to the increase in re-adsorption amount of SDS. This can arise from the ion suppression effect of SDS. The regeneration times under the $1 \mathrm{mM}$ SDS condition were strongly affected by the $\mathrm{HClO}_{4}$ concentration. Especially in the case of the C18 column with $1 \mathrm{mM}$ $\mathrm{SDS} / 10 \mathrm{mM} \mathrm{HClO}_{4}$ eluent, the appearance of the system peak at the column reequilibration point was not observed even after allowing much time to confirm it. With $10 \mathrm{mM}$ SDS, the regeneration time was almost independent of the acid concentration. Therefore 5-10 $\mathrm{mM}$ of SDS was considered to be adequate for the composition of mobile phase A in practical use.

On the other hand, the retention of amino acids was decreased, in all cases, as the concentration of $\mathrm{HClO}_{4}$ was decreased. In low acid concentration, the amounts of SDS in the stationary phases also decreased owing to an insufficient SDS ion suppression effect, as shown in Fig. 2, so that the cation-exchange site itself must be decreased; it thus follows that the apparent ion-exchange capacity is decreased. Consequently in order to reduce the chromatographic cycle time, it is desirable to use a relatively lower hydrophobic reversed-phase column with an eluent in lower acid concentration and higher SDS concentration.

In a previous study ${ }^{12}$, a $15 \mathrm{~cm}$-long $\mathrm{C} 4$ column with

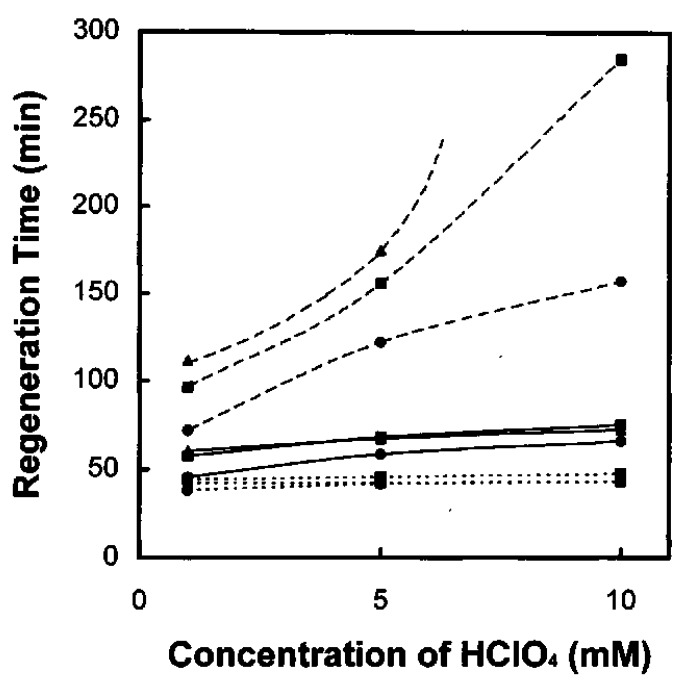

Fig. 2 Relationships between $\mathrm{HClO}_{4}$ concentration in mobile phase and regeneration time. Symbols represent column type as: $\bullet, C 4 ; \square, C 8 ; \Delta, C 18$, and lines represent SDS concentration as: broken, $1 \mathrm{mM}$; solid, $5 \mathrm{mM}$; dotted, $10 \mathrm{mM}$.

$5 \mathrm{mM}$ SDS $/ 10 \mathrm{mM} \mathrm{HClO}_{4}$ as the mobile phase $\mathrm{A}$ has been successfully used to obtain satisfactory results in amino acid separation. In this study, a $25 \mathrm{~cm}$-long C4 column was examined under the same elution conditions in expectation of a higher resolution. Even though its theoretical plate number was higher, the anticipated better separation of the amino acids was not observed; even more, the $25 \mathrm{~cm}$ column required a $60 \%$ longer cycle time than the $15 \mathrm{~cm}$ column did. The pressure drop was also larger in proportion to the column length. The use of $25 \mathrm{~cm}$ columns seems to be disadvantageous for gradient ion-pair chromatography.

From the point of view of amino acid separation, some combinations of column type and mobile phase A were adequate: these were the $\mathrm{C} 4$ column and $5 \mathrm{mM}$ SDS/ $10 \mathrm{mM} \mathrm{HClO}_{4}$; the $\mathrm{C} 8$ column and $5 \mathrm{mM} \mathrm{SDS} / 5 \mathrm{mM}$ $\mathrm{HClO}_{4}$; and the $\mathrm{Cl} 8$ column and $1 \mathrm{mM}$ SDS $/ 5 \mathrm{mM}$ $\mathrm{HClO}_{4}$. Figure 3 shows a chromatogram of standard amino acids by the $\mathrm{C} 4$ column system (acetonitrile gradient: $15 \rightarrow 35 \%$ in $50 \mathrm{~min}$ at flow rate of $1 \mathrm{ml} / \mathrm{min}$ ), where the cycle time was about $108 \mathrm{~min}$. The cycle times of $\mathrm{C} 8$ and $\mathrm{C} 18$ column systems were 133 and $128 \mathrm{~min}$ under the same elution condition, respectively. In both the $\mathrm{C} 8$ and $\mathrm{C} 18$ column systems, different gradient curves were required to elute all amino acids in $55 \mathrm{~min}$ because of the higher column hydrophobicity.

The $\mathrm{C} 8$ column system by the dual-mode ${ }^{12}$ gradient elution (mobile phase B, acetonitrile: $15 \rightarrow 40 \%$ in $60 \mathrm{~min}$; flow rate: $1 \rightarrow 2 \mathrm{ml} / \mathrm{min}$ in $60 \mathrm{~min}$ ) provided a good separation, but required $100 \mathrm{~min}$ for the cycle time. In contrast, the cycle time of the $\mathrm{C} 4$ column system by the dual mode gradient elution (B: $15 \rightarrow 35 \%$ in $50 \mathrm{~min}$; flow rate: $1 \rightarrow 2 \mathrm{ml} / \mathrm{min}$ in $50 \mathrm{~min}$ ) was $80 \mathrm{~min} .^{12}$ In the $\mathrm{C18}$ column system with the acetonitrile gradient at a con- 


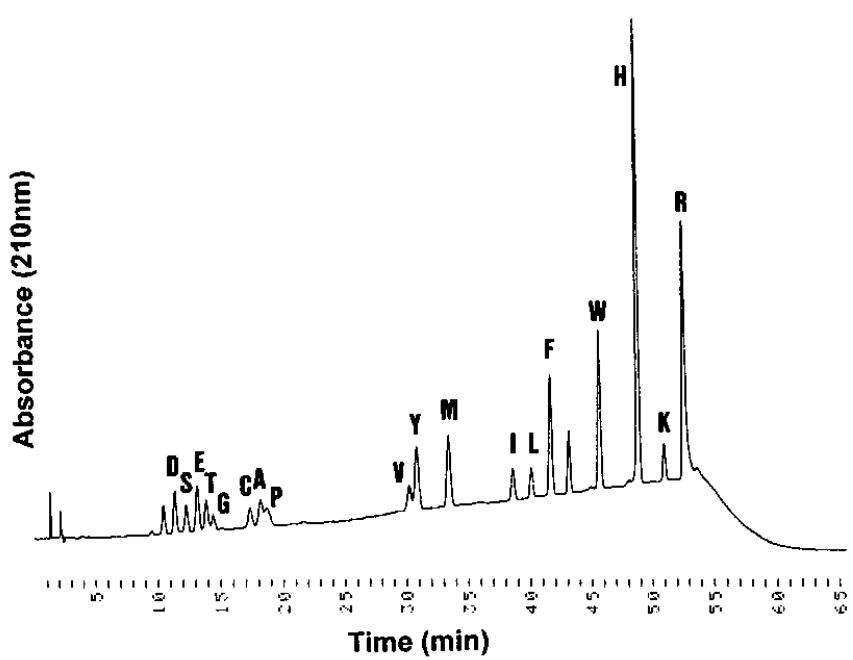

Fig. 3 Chromatogram of standard amino acids by $\mathrm{C} 4$ column with mobile phase A: $5 \mathrm{mM}$ SDS $/ 10 \mathrm{mM} \mathrm{HClO}_{4}$ and $\mathrm{B}$ : acetonitrile gradient elution system at $1 \mathrm{ml} / \mathrm{min}$. Symbols are one letter symbols of amino acids. Concentrations: $\mathrm{H}=100 \mu \mathrm{M} ; \mathrm{Y}, \mathrm{F}=25 \mu \mathrm{M} ; \mathrm{W}=10 \mu \mathrm{M} ;$ others $=1 \mathrm{mM}$. Sample size: $20 \mu \mathrm{l}$. Detection: UV $210 \mathrm{~nm}$.

stant flow rate of $1 \mathrm{ml} / \mathrm{min}$, an excellent resolution especially for hydrophilic amino acids was obtained, but the system was impractical for routine analysis because of its unreasonably long regeneration time, about $3 \mathrm{~h}$ as shown in Fig. 2. In a different $\mathrm{Cl} 8$ column system with $5 \mathrm{mM} \mathrm{SDS} / 10 \mathrm{mM} \mathrm{HClO}_{4}$ by dual-mode gradient elution (B: $15 \rightarrow 40 \%$ in $50 \mathrm{~min}$; flow rate: $1 \rightarrow 2 \mathrm{ml} / \mathrm{min}$ in $50 \mathrm{~min}$ ), the cycle time was $95 \mathrm{~min}$, which was $5 \mathrm{~min}$ shorter than the $\mathrm{C} 8$ system. The inconsistency of these results is considered to be also due to the soak problem between the stationary and mobile phases as mentioned above. Thus we can conclude that the $\mathrm{C} 4$ column system with a dual-mode gradient elution is the best choice for ion-pair chromatography of amino acids, as described in the previous paper. ${ }^{12}$

The concentration of SDS should be increased in order to reduce the cycle time. Under such conditions, however, further improvement in amino acid separation can not be expected because the acetonitrile concentration must be increased in proportion to the SDS concentration. We consider therefore that the retention time of each amino acid would be relatively unchanged by changing SDS concentration.

\section{Effect of alkyl chain length of ion-pairing agent}

The use of ion-pairing agent in high concentration is considered to be effective in reducing the chromatographic cycle time. Additionally, another ion-pairing agent of a different alkyl chain length may provide another selectivity for amino acids. Thus the retention behavior of amino acids was investigated by using sodium pentanesulfonate and sodium octanesulfonate in high concentrations.

Since the adsorption power of SPS, even in con-

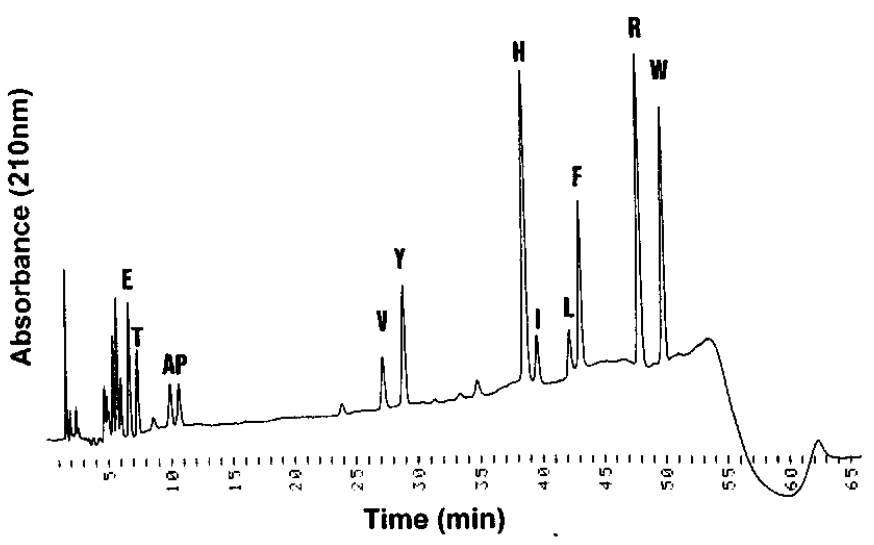

Fig. 4 Chromatogram of standard amino acids by $\mathrm{C} 4$ column with mobile phase A: $100 \mathrm{mM}$ SOS $/ 10 \mathrm{mM} \mathrm{HClO}_{4}$ and B: acetonitrile gradient elution system at $1 \mathrm{ml} / \mathrm{min}$. Other conditions: as in Fig. 3.

centration of $200 \mathrm{mM}$ without organic solvent, was insufficient for retaining amino acid cations on the reversed-phase column owing to the anion's weak hydrophobicity, further investigation was not carried out.

On the one hand, SOS showed moderate ion-pairing power for amino acids. Relationships between the nominal carbon loadings and the re-adsorption amounts of SOS were relatively similar to those of SDS shown in Fig. 1. Under the $10 \mathrm{mM}$-SOS condition, the retention time of the system peak for the C18 column was also shorter than that for the $\mathrm{C} 8$ column. The regeneration time was allowed to shorten according as the SOS concentration increased from 10 to $100 \mathrm{mM}$. Under the $100 \mathrm{mM}$-SOS condition, the cycle time was to be independent of column hydrophobicity, showing almost the same time as $65 \mathrm{~min}$, which corresponded to a 40-min time saving compared with the case using the $\mathrm{C} 4$ column with $5 \mathrm{mM}$ SDS mentioned above. The chromatographic reproducibility was good, showing a relative standard deviation of under $0.9 \%$ for the retention time of each amino acid; however, the chromatographic separation was insufficient compared with the results under the SDS gradient elution condition. Under the $100 \mathrm{mM}-\mathrm{SOS}$ condition, the C18 column was rather adequate for separating acidic and neutral-hydrophilic amino acids and the $\mathrm{C} 4$ column was adequate for basic and neutral-hydrophobic amino acids.

Figure 4 shows a chromatogram of standard amino acids using the $\mathrm{C} 4$ column with $100 \mathrm{mM}$ SOS $/ 10 \mathrm{mM}$ $\mathrm{HClO}_{4}$ as mobile phase A. A linear gradient elution of acetonitrile (mobile phase B) from 0 to $25 \%(\mathrm{v} / \mathrm{v})$ was adequate. Since the retention of hydrophilic amino acids was weak even under such water-rich conditions, only a poor separation among them was observed, as seen between 4 and $8 \mathrm{~min}$ of retention time. In contrast, a satisfactory separation among hydrophobic and basic amino acids was obtained. The eluting order of the amino acids by the SOS system was much different from 
those by the SDS system (Fig. 3) which was similar to those by the usual cation-exchange chromatography. The retention of hydrophobic amino acids such as Ile, Leu, Phe, and Trp was relatively stronger than that of the basic ones such as His, Lys, and Arg. The result may indicate a difference in the retention mechanism of the amino acids in ion-pair HPLC. It is considered that the retention mechanism on the SDS system is dominated by an ion-exchange interaction between the amino acid cation in the mobile phase and the SDS anion adsorbed in the stationary phase, and that on the SOS system is dominated mainly by the hydrophobic interaction due to the distribution of neutral ion pairs formed between amino acid cations and SOS anions. ${ }^{14}$

Although the use of SOS as an ion-pairing agent for amino acids is favorable in terms of reducing the cycle time, its high concentration in the eluent can give the following disadvantages for practical routine use: providing unexpected ghost peaks due to impurities; increasing background noise and baseline drift during gradient elution; reducing column life by accumulation of undesorbable impurities; requiring high running cost because of the need for an expensive reagent. Consequently, the SOS ion-pair HPLC system seems to be impractical for routine analyses.

In conclusion, the combination of a $15 \mathrm{~cm}$-long butylsilica reversed-phase column and a $5 \mathrm{mM}$ SDS/ $10 \mathrm{mM}$ $\mathrm{HClO}_{4}$-acetonitrile binary gradient elution system is the best choice for the gradient ion-pair chromatography of amino acids. Although the chromatographic cycle time is dependent on the column product lot, the relative separation among amino acids is considered to be unchanged. The butyl-silica column used is durable enough to have a significant column lifetime to be able to perform replicate chromatography. If necessary, UV detection and/or postcolumn fluorescence detection ${ }^{12}$ are available. The developed high-pressure binary gradient ion-pair chromatography system using conventional HPLC equipment will show an excellent costperformance ratio for amino acid analysis, and is considered to be useful for clinical, food science, and chemical research laboratories where they do not have expensive amino acid analyzers.

\section{References}

1. M. Roth, Anal. Chem., 43, 880 (1971).

2. M. Roth and A. Hampai, J. Chromatogr., 83, 353 (1973).

3. J. R. Benson and P. E. Hare, Proc. Natl. Acad. Sci. USA, 72, 619 (1975).

4. D. W. Hill, F. H. Walters, T. D. Wilson and J. D. Stuart, Anal. Chem., 51, 1338 (1979).

5. P. Lindroth and K. Mopper, Anal. Chem., 51, 1667 (1979).

6. B. R. Larsen and F. G. West, J. Chromatogr. Sci., 19, 259 (1981).

7. M. K. Radjai and R. T. Hatch, J. Chromatogr., 196, 319 (1980).

8. T. Hayashi, H. Tsuchiya and H. Naruse, J. Chromatogr., 274, 318 (1983).

9. T. Hayashi, M. Komaki, H. Tsuchiya, F. Matsuda and H. Naruse, Bunseki Kagaku, 35, 949 (1986).

10. L. R. Snyder, J. L. Glajch and J. J. Kirkland, "Practical HPLC Method Development", p. 71, John Wiley \& Sons, New York, 1988.

11. M. Patthy, J. Chromatogr., 592, 143 (1992).

12. Y. Yokoyama, O. Ozaki and H. Sato, J. Chromatogr. A, 739, 333 (1996).

13. J. H. Knox and R. A. Hartwick, J. Chromatogr., 204, 3 (1981).

14. B. A. Bidlingmeyer, J. Chromatogr. Sci., 18, 525 (1980).

(Received July 11, 1997)

(Accepted September 10, 1997) 